Dhaka Univ. J. Sci. 61(1): 139-144, 2013 (January)

\title{
An Analytic Treatment of the Falkner-Skan Boundary Layer Equation Mohammad Riazuddin Molla
}

\author{
Department of Mathematics, University of Dhaka, Dhaka-1000, Bangladesh
}

E-mail:mriaz_math@yahoo.com

Received on 06.08.2012. Accepted for Published on 13.10.2012

\begin{abstract}
The existence and uniqueness of a positive solution of a singular nonlinear boundary value problem formulated from the Falkner-Skan boundary layer equation, are studied. The constructive method such as the method of upper and lower solutions is used to show the existence and uniqueness of a positive solution.
\end{abstract}

Keywords: Positive upper and lower solutions; Falkner-Skan boundary layer equation; Crocoo variables.

\section{Introduction}

The differential equation

$$
f^{\prime \prime \prime}+\alpha f f^{\prime \prime}+\beta\left(1-f^{\prime 2}\right)=0,
$$

with boundary conditions

$$
\begin{gathered}
f(\eta)=f^{\prime}(\eta)=0 \text { at } \eta=0 \\
\text { and } f^{\prime}(\eta) \rightarrow 1 \text { as } \eta \rightarrow \infty .
\end{gathered}
$$

is known as Falkner-Skan boundary layer problem. Shin ${ }^{1}$ studied the differential equation (1.1) for $\alpha=1$ and $\beta=0.5$ with boundary conditions (1.2). For $\alpha=1$ and $\beta=0.5$, the differential equation (1.1) with boundary conditions (1.2) represents the Homann flow ${ }^{1}$. For $\alpha=1$ and $\beta$ negative, the differential equation (1.1) with boundary conditions (1.2) represents decelerating flow ${ }^{2}$. In this article the analysis is made for $\alpha=1$ and $\beta=-k$, where $0<k \leq 1$.

For $\alpha=1$ and $\beta=-k$, equation (1.1) takes the form

$$
f^{\prime \prime \prime}+f f^{\prime \prime}-k\left(1-f^{\prime 2}\right)=0
$$

with boundary conditions (1.2).

Let Shear stress $y\left(=f^{\prime \prime}(n)\right)$ as the dependent variable and tangential velocity $x\left(=f^{\prime}(n)\right)$ as the independent variable. The quantities $x$ and $y$ are called Crocco variables.

Now

$$
\begin{aligned}
& y^{\prime}=\frac{d y}{d x}=\frac{\frac{d y}{d \eta}}{\frac{d x}{d \eta}}=\frac{\frac{d}{d \eta}\left(f^{\prime \prime}\right)}{\frac{d}{d \eta}\left(f^{\prime}\right)}=\frac{f^{\prime \prime \prime}}{f^{\prime \prime}} \text {, which implies } \\
& y^{\prime} f^{\prime \prime}=f^{\prime \prime \prime}
\end{aligned}
$$

Differentiating (1.4) with respect to $\eta$ and simplifying we get

$$
f^{i v}=y^{2} y^{\prime \prime}+y\left(y^{\prime}\right)^{2}
$$

As before differentiating (1.3) with respect to $\eta$ one gets

$$
f^{i v}+(2 k+1) f f^{\prime \prime}+f f^{\prime \prime \prime}=0 \text {. }
$$

This gives

$$
y^{2} y^{\prime \prime}+y\left(y^{\prime}\right)^{2}+(2 k+1) x y+y^{\prime}\left[k\left(1-x^{2}\right)-y y^{\prime}\right]=0
$$

and finally we get

$$
\begin{gathered}
y^{2} y^{\prime \prime}+(2 k+1) x y+k\left(1-x^{2}\right) y^{\prime}=0 . \\
\text { Let } 2 k+1=k_{1} \text { for } 0<k \leq 1 .
\end{gathered}
$$

For $\quad \eta=0$,we get $\quad x=f^{\prime}(0)=0, \quad$ and $\eta \rightarrow \infty, \quad x \rightarrow 1$. Hence the transformation $x=f^{\prime}(\eta)$ maps the $\eta$-interval $[0, \infty)$ into the $x$-interval $[0,1)$.

Assuming a monotonic dependence of $x$ on $\eta$ we get $x=0$ implies $\eta=0$ and $x \rightarrow 1$ implies $\eta \rightarrow \infty$.

Then

$$
y(1)=\operatorname{Lim}_{\eta \rightarrow \infty} f^{\prime \prime}(\eta)=\operatorname{Lim}_{\Delta \eta \rightarrow 0} \frac{f^{\prime}(\infty+\Delta \eta)-f^{\prime}(\infty)}{\Delta \eta}=0 .
$$

That is; $y(1)=0$.

Again, from (1.3) and (1.4), we get

$$
y^{\prime}(x)=\frac{k}{y}\left(1-x^{2}\right)-f(\eta) .
$$

Hence $y^{\prime}(0)=\frac{k}{y(0)}(1-0)-f(0)=\frac{k}{y(0)}$,implies $y^{\prime}(0) y(0)=k$.

Thus by letting $y\left(=f^{\prime \prime}(n)\right)$ and $x\left(=f^{\prime}(n)\right)$, the boundary layer problem of equation (1.3) with boundary conditions (1.2) can be written as

*Author for correspondence 


$$
\left.\begin{array}{l}
y^{2} y^{\prime \prime}+k_{1} x y+k\left(1-x^{2}\right) y^{\prime}=0, \quad 0 \leq x<1 \\
y^{\prime}(0) y(0)=k, \quad y(1)=0
\end{array}\right\}
$$

Assume $y(0)>0$ because we discuss the existence and uniqueness of a positive solution of a singular nonlinear boundary value problem.

$$
\text { Here } y(0)=f^{\prime \prime}(0) \text {. }
$$

The value of $f^{\prime \prime}(0)$ is still unknown ${ }^{2}$. We assume $f(\eta)=\eta-\ln (1+\eta)$,

which satisfies the three boundary conditions of (1.2).

From (1.7) one gets easily that

$$
\begin{gathered}
f^{\prime \prime}(\eta)=\frac{1}{(\eta+1)^{2}}, \text { from which we get } \\
f^{\prime \prime}(0)=1 .
\end{gathered}
$$

Then the boundary value problem (1.6) becomes

$$
\left.\begin{array}{l}
y^{2} y^{\prime \prime}+k_{1} x y+k\left(1-x^{2}\right) y^{\prime}=0, \quad 0 \leq x<1 \\
y^{\prime}(0)=k, \quad y(1)=0
\end{array}\right\}
$$

For $\alpha=1$ and $\beta=0.5$, equation (1.1) with boundary conditions (1.2) takes the form

$$
\left.\begin{array}{l}
y^{2} y^{\prime \prime}-\frac{1}{2}\left(1-x^{2}\right) y^{\prime}=0, \quad 0 \leq x<1 \\
y^{\prime}(0)=-0.5, y(1)=0
\end{array}\right\}
$$

and its positive solution has been studied by $\mathrm{Shin}^{1}$ using a constructive method such as the method of upper and lower solutions.

The objective of this paper is to establish the existence and uniqueness of a positive solution of singular non-linear boundary value problem of the form(1.8) using a constructive method such as the method of upper and lower solutions.

Definition1.1: We call a function $\alpha_{1} \in C^{2}[0,1]$ a positive upper solution of (1.8) if

$$
\begin{gathered}
\alpha_{1}>0 \text { on }(0,1) \\
\alpha_{1}^{2} \alpha_{1}^{\prime \prime}+k_{1} x \alpha_{1}+k\left(1-x^{2}\right) \alpha_{1}^{\prime} \leq 0 \text { on }(0,1) \\
\alpha_{1}^{\prime}(0) \leq k \text { and } \alpha_{1}(1) \geq 0 .
\end{gathered}
$$

Definition1.2: We call a function $\alpha_{2} \in C^{2}[0,1]$ a positive lower solution of (1.8), if

$$
\begin{gathered}
\alpha_{2}>0 \text { on }(0,1) \\
\alpha_{2}^{2} \alpha_{2}^{\prime \prime}+k_{1} x \alpha_{2}+k\left(1-x^{2}\right) \alpha_{2}^{\prime} \geq 0 \text { on }(0,1) \\
\alpha_{2}^{\prime}(0) \geq k \text { and } \alpha_{2}(1) \leq 0 .
\end{gathered}
$$

Similar definitions hold for positive upper and lower solutions of a perturbation of (2.1) which will be given in the following section.

Definition1.3: We call a function $y \in C[0,1] \cap C^{2}[0,1)$ a positive solution of (1.8) if

$$
\begin{gathered}
y>0 \text { on }(0,1) \\
y^{2} y^{\prime \prime}+k_{1} x y+k\left(1-x^{2}\right) y^{\prime}=0 \text { on }(0,1) \\
y^{\prime}(0)=k \text { and } y(1)=0 .
\end{gathered}
$$

\section{Existence of a Unique Positive Solution}

For each $p \geq 1$, we consider the nonlinear boundary value problem

$$
\left.\begin{array}{l}
y^{2} y^{\prime \prime}+k_{1} x y+k\left(1-x^{2}\right) y^{\prime}=0, \quad 0 \leq x<1 \\
y^{\prime}(0)=k, \quad y(1)=\frac{1}{p}
\end{array}\right\}
$$

which is a perturbation of (1.8).

To prove the existence of positive solution of (1.8) we establish the existence of positive solution of (2.1).

Lemma 2.1: $y_{u p}(x)=3 \ln (2-x)+(k+1) x+\frac{1}{p}$ is a positive upper solution of (2.1), for each $p \geq 1$ and $0<k \leq 1$.

Proof: It is clear that

$y_{u p}(x)>0$ on $(0,1), y_{u p}^{\prime}(0)=-\frac{3}{2}+k+1$, which can be written as

$y_{u p}^{\prime}(0)=-\frac{3}{2}+k+1 \leq k, y_{u p}(1)=k+1+\frac{1}{p}$, which can

be written as $y_{u p}(1)=k+1+\frac{1}{p} \geq \frac{1}{p}$ and

$y_{u p}^{2} y_{u p}^{\prime \prime}+k_{1} x y_{u p}+k\left(1-x^{2}\right) y_{u p}^{\prime}=$

$\frac{-3}{(2-x)^{2}}\left[3 \ln (2-x)+(k+1) x+\frac{1}{p}\right]^{2}+$

$$
\begin{aligned}
& k_{1} x\left[3 \ln (2-x)+(k+1) x+\frac{1}{p}\right]+ \\
& k\left(1-x^{2}\right)\left[-\frac{3}{(2-x)}+k+1\right] \leq 0,
\end{aligned}
$$


for $0<x<1, p \geq 1$ and $0<k \leq 1$.

Thus $y_{u p}$ is a positive upper solution of (2.1).

Lemma 2.2:

$y_{l p}(x)=k x^{4} \ln (2-x)+k x(1-x)^{2}+\frac{1}{p+\frac{50}{k}}$

is a positive lower solution of (2.1), for each $p \geq 1$ and $0<k \leq 1$.

Proof: It is clear that $y_{l p}(x)>0$, on $(0,1)$ and $y_{l p}^{\prime}(0)=k$, which can be written as

$y_{l p}^{\prime}(0)=k \geq k, y_{l p}(1)=\frac{1}{p+\frac{50}{k}}$, which can be written

as $y_{l p}(1)=\frac{1}{p+\frac{50}{k}} \leq \frac{1}{p}$.

Also

$y_{l p}^{2} y_{l p}^{\prime \prime}+k_{1} x y_{l p}+k\left(1-x^{2}\right) y_{l p}^{\prime} \geq 0$, for

$0<x<1, p \geq 1$ and $0<k \leq 1$.

Thus $y_{l p}$ is a positive lower solution of (2.1).

Hence we can formulate the following Lemma2.3 as an application of Schauder's Fixed Point Theorem ${ }^{3}$.

Lemma 2.3:For any $p \geq 1$, there exists a positive solution $y_{p} \in C^{2}[0,1] \quad$ of the problem (2.1)such that $y_{l p} \leq y_{p} \leq y_{u p}$ on $0 \leq x \leq 1$, where $y_{l p}$ and $y_{u p}$ are as given in Lemma 2.2 and Lemma 2.1 respectively.

Lemma 2.4:If $y_{p}$ is a positive solution of (2.1) and $y_{p}^{\prime}(x)>0$ then $0<y_{p}^{\prime}(x)<k$ on $(0,1]$ for $p \geq 1$ and $0<k \leq 1$.

Proof: Since $y_{p}$ is positive solution of (2.1), we get

$$
y_{p}^{2} y_{p}^{\prime \prime}+k_{1} x y_{p}+k\left(1-x^{2}\right) y_{p}^{\prime}=0 .
$$

This gives

$y_{p}^{2} y_{p}^{\prime \prime} \leq-k\left(1-x^{2}\right) y_{p}^{\prime}$, which implies

$$
\frac{y_{p}^{\prime \prime}}{y_{p}^{\prime}} \leq-\frac{k\left(1-x^{2}\right)}{y_{p}^{2}}<0 .
$$

Integrating from 0 to $x$, we get $\ln \left(y_{p}^{\prime}(x)\right)-\ln \left(y_{p}^{\prime}(0)\right)<0, \quad$ it $\quad$ follows that $0<y_{p}^{\prime}(x)<1$ on $(0,1]$.

Lemma 2.5: If $y_{p}$ is a positive solution of (2.1) and $y_{p}^{\prime}(x)<0$ then $y_{p}^{\prime}(x)<k$ on $(0,1]$, for $p \geq 1$ and $0<k \leq 1$.

Proof: Since $y_{p}$ is positive solution of (2.1), we get

$$
y_{p}^{2} y_{p}^{\prime \prime}+k_{1} x y_{p}+k\left(1-x^{2}\right) y_{p}^{\prime}=0 .
$$

This gives

$$
\begin{array}{r}
y_{p}^{2} y_{p}^{\prime \prime} \leq-k\left(1-x^{2}\right) y_{p}^{\prime}, \text { which implies } \\
\frac{y_{p}^{\prime \prime}}{-y_{p}^{\prime}} \leq \frac{k\left(1-x^{2}\right)}{y_{p}^{2}} .
\end{array}
$$

For $x \in(0,1], \frac{y_{p}^{\prime \prime}}{-y_{p}^{\prime}} \leq \frac{k\left(1-x^{2}\right)}{y_{p}^{2}}$ is true if $\frac{y_{p}^{\prime \prime}}{-y_{p}^{\prime}}<0$.

Now $\frac{y_{p}^{\prime \prime}}{-y_{p}^{\prime}}<0$ gives $y_{p}^{\prime \prime}<0$, because $-y_{p}^{\prime}(x)>0$.

Integrating $y_{p}^{\prime \prime}<0 \quad$ from $0 \quad$ to $x$, we get $y_{p}^{\prime}(x)-y_{p}^{\prime}(0)<0$, it follows that $y_{p}^{\prime}(x)<k$ on $(0$, 1], because $y_{p}^{\prime}(0)=k$.

Lemma 2.6: If $y_{1}$ and $y_{2}$ are two positive solutions of (2.1) then $y_{1}=y_{2}$, for $p \geq 1$ and $0<k \leq 1$.

Proof: Since $y_{1}$ and $y_{2}$ are two positive solutions of (2.1), we get

$y_{1}^{\prime \prime}=-\frac{k_{1} x}{y_{1}}-\frac{k\left(1-x^{2}\right) y_{1}^{\prime}}{y_{1}^{2}}$ and

$y_{2}^{\prime \prime}=-\frac{k_{1} x}{y_{2}}-\frac{k\left(1-x^{2}\right) y_{2}^{\prime}}{y_{2}^{2}}$.

Suppose that there exists an $\eta \in(0,1]$ such that $y_{1}(\eta) \neq y_{2}(\eta)$. 
If $y_{1}(0)=y_{2}(0)$, then by the uniqueness theorem of the initial value problem, we get $y_{1}=y_{2}$, which is a contradiction. Therefore, without loss of generality, we may assume that $y_{1}(0)<y_{2}(0)$.

Since $y_{1}(1)<y_{2}(1)$ by the continuity of $y_{1}-y_{2}$, there exists a $\quad \xi \in(0,1]$ such that $y_{1}(\xi)=y_{2}(\xi)$ and $\quad y_{1}(x)<y_{2}(x)$ on $[0, \xi)$.

So the function $\phi(x)=y_{2}(x)-y_{1}(x)$ has a maximum at $x=0$ or at the interior of $[0, \xi]$.

If the function $\phi(x)$ takes its minimum at $x=0$, then we obtain $\phi^{\prime \prime}(0)>0$.

Now,

$\phi^{\prime \prime}(0)=\operatorname{Lim}_{x \rightarrow 0^{+}} \frac{\phi^{\prime}(x)-\phi^{\prime}(0)}{x}>0$.

It follows that $\phi^{\prime}(x)>\phi^{\prime}(0)=0$ near $x=0$ and hence $\phi(x)>\phi(0)$ near $x=0$.

This is a contradiction.

Hence $\phi(x)$ does not have a minimum at $x=0$.

If the function $\phi(x)$ takes its maximum at the interior of $[0, \xi]$, then there exists a $\xi_{1} \in(0, \xi)$ such that $\phi^{\prime}\left(\xi_{1}\right)=0$ and $\phi^{\prime \prime}\left(\xi_{1}\right)<0$.

But since $y_{1}\left(\xi_{1}\right), y_{2}\left(\xi_{1}\right)$ are solutions of (2.1), $y_{1}^{\prime}\left(\xi_{1}\right)<k \quad, \quad y_{1}\left(\xi_{1}\right)<y_{2}\left(\xi_{1}\right) \quad$ and $0<y_{1}^{\prime}\left(\xi_{1}\right)<k$,we obtain,

$\phi^{\prime \prime}\left(\xi_{1}\right)=y_{2}^{\prime \prime}\left(\xi_{1}\right)-y_{1}^{\prime \prime}\left(\xi_{1}\right)$

$$
\begin{aligned}
& =\frac{k_{1} \xi_{1}}{y_{1}}+\frac{k\left(1-\xi_{1}^{2}\right) y_{1}^{\prime}}{y_{1}^{2}}-\frac{k_{1} \xi_{1}}{y_{2}}-\frac{k\left(1-\xi_{1}^{2}\right) y_{2}^{\prime}}{y_{2}^{2}} \\
& =k_{1} \xi_{1}\left(\frac{1}{y_{1}}-\frac{1}{y_{2}}\right)+k\left(1-\xi_{1}^{2}\right) y_{1}^{\prime}\left(\frac{1}{y_{1}^{2}}-\frac{1}{y_{2}^{2}}\right)>0,
\end{aligned}
$$

which again leads to a contradiction. Hence $\phi(x)$ does not have a maximum at the interior of $[0, \xi]$.

This implies that $y_{1}=y_{2}$.

Lemma 2.7:If $y_{p}$ is a positive solution of (2.1) for each $p \geq 1$ and $0<k \leq 1$, then we obtain $y_{p}^{\prime}(x) \geq\left[k-\int_{0}^{x} \frac{k_{1} s y_{l p}(s)+k\left(1-s^{2}\right) y_{p}^{\prime}(s)}{y_{l p}^{2}(s)} d s\right]$

$[0,1)$

Proof: Let $y_{l p}$ be a positive lower solution of (2.1). Since $y_{p} \in C^{2}[0,1]$ is a positive solution of $(2.1)$, we obtain $y_{l p} \leq y_{p}$ and $y_{p}^{2} y_{p}^{\prime \prime}+k_{1} x y_{p}+k\left(1-x^{2}\right) y_{p}^{\prime}=0$.

Now

$$
\begin{aligned}
0=y_{p}^{\prime \prime}+\frac{k_{1} x}{y_{p}}+\frac{k\left(1-x^{2}\right) y_{p}^{\prime}}{y_{p}^{2}} & \leq y_{p}^{\prime \prime}+\frac{k_{1} x}{y_{l p}} \\
& +k\left(1-x^{2}\right) \frac{y_{p}^{\prime}}{y_{l p}^{2}}
\end{aligned}
$$

or, $y_{p}^{\prime \prime}+\frac{k_{1} x}{y_{l p}}+\frac{k\left(1-x^{2}\right) y_{p}^{\prime}}{y_{l p}^{2}} \geq 0$

or, $y_{p}^{\prime \prime} \geq-\left[\frac{k_{1} x y_{l p}+k\left(1-x^{2}\right) y_{p}^{\prime}}{y_{l p}^{2}}\right]$

Integrating from 0 to $x$, we obtain

$y_{p}^{\prime}(x) \geq\left[k-\int_{0}^{x} \frac{k_{1} s y_{l p}(s)+k\left(1-s^{2}\right) y_{p}^{\prime}(s)}{y_{l p}^{2}(s)} d s\right]$ on

$[0,1)$, because $y_{p}^{\prime}(0)=k$.

Lemma 2.8: If $p_{1}>p_{2} \geq 1$ and $y_{p_{1}}, y_{p_{2}}$ are positive solutions of (2.1) respectively, then we have $0<y_{p_{1}}(x)<y_{p_{2}}(x)$ on $(0,1)$ and

$$
y_{p_{1}}^{\prime}(x)<y_{p_{2}}^{\prime}(x)<k \text { on }(0,1] \text {. }
$$

Proof: It is clear from the fact that $y_{p_{2}}$ is a positive upper solution of (2.1) and so

$$
y_{p_{1}}(x) \leq y_{p_{2}}(x) \text { on }[0,1] \text {. }
$$

If $y_{p_{1}}(0)=y_{p_{2}}(0)$, then by the uniqueness theorem of the initial value problem we obtain

$$
y_{p_{1}}(x) \equiv y_{p_{2}}(x) \text {, }
$$

which is a contradiction. So we may assume that $0<y_{p_{1}}(0)<y_{p_{2}}(0)$. Then we have

$$
y_{p_{2}}^{\prime \prime}(0)-y_{p_{1}}^{\prime \prime}(0)=k y_{p_{1}}^{\prime}(0)\left(\frac{1}{y_{p_{1}}^{2}(0)}-\frac{1}{y_{p_{2}}^{2}(0)}\right)>0
$$


which implies that

$y_{p_{2}}^{\prime}(x)-y_{p_{1}}^{\prime}(x)>y_{p_{2}}^{\prime}(0)-y_{p_{1}}^{\prime}(0)=-k+k=0$,

$y_{p_{2}}(x)-y_{p_{1}}(x)>y_{p_{2}}(0)-y_{p_{1}}(0)>0$, for $x$ near to

0 .

If there exists a $\quad \xi \in(0,1]$ such that $y_{p_{2}}^{\prime}(\xi)-y_{p_{1}}^{\prime}(\xi)=0$ and $y_{p_{2}}^{\prime}(x)-y_{p_{1}}^{\prime}(x)>0$, $0<x<1$.

Then we obtain

$$
\begin{aligned}
& y_{p_{2}}^{\prime \prime}(\xi)-y_{p_{1}}^{\prime \prime}(\xi)=k_{1} \xi\left(\frac{1}{y_{p_{1}}(\xi)}-\frac{1}{y_{p_{2}}(\xi)}\right)+ \\
& k\left(1-\xi^{2}\right) y_{p_{1}}^{\prime}(\xi)\left(\frac{1}{y_{p_{1}}^{2}(\xi)}-\frac{1}{y_{p_{2}}^{2}(\xi)}\right)>0,
\end{aligned}
$$$$
\text { because } y_{p_{1}}^{\prime}(\xi)<k, 0<y_{p_{1}}^{\prime}(\xi)<k \text { and }
$$$$
y_{p_{2}}(\xi)-y_{p_{1}}(\xi)>0 \text {. }
$$

Hence we have

$y_{p_{2}}^{\prime}(x)-y_{p_{1}}^{\prime}(x)<y_{p_{2}}^{\prime}(\xi)-y_{p_{1}}^{\prime}(\xi)=0,0<x<\xi$, which is a contradiction. This completes the proof.

Theorem 2.9: (Existence):If $y_{p}$ is the positive solution of (2.1) for each $p=1,2,3 \ldots \ldots \ldots$, then the sequence $\left\{y_{p}\right\}$ converges to a positive solution $y$ of (1.8).

Proof: To prove this theorem, we prove the following steps:

$$
\begin{array}{ll}
\text { Step 1. } & y_{p} \rightarrow y \text { as } p \rightarrow \infty . \\
\text { Step 2. } y \in C[0,1] \cap C^{2}[0,1) . \\
\text { Step 3. } y \text { is a positive solution of (1.8). }
\end{array}
$$

Our first step is to show that $y_{p} \rightarrow y$ as $p \rightarrow \infty$. We know that the sequence $\left\{y_{p}\right\}$ is monotone decreasing $\begin{array}{llll}\text { in } p & \text { and } & \text { bounded below by } \\ k x^{4} \ln (2-x)+k x(1-x)^{2}+\frac{1}{p+\frac{50}{k}} & \end{array}$

Therefore, $y_{p} \rightarrow y$ as $p \rightarrow \infty$ and $y(x) \geq k x^{4} \ln (2-x)+k x(1-x)^{2}$ on $(0,1]$.
Also from Lemma 2.7 and Lemma 2.8, we know that the sequence $\left\{y_{p}^{\prime}\right\}$ is monotone decreasing in $p$ and bounded below by

$\left[k-\int_{0}^{x} \frac{k_{1} s y_{l p}(s)+k\left(1-s^{2}\right) y_{p}^{\prime}(s)}{y_{l p}^{2}(s)} d s\right]$ on $[0,1)$.

Therefore, $y_{p}^{\prime} \rightarrow y^{\prime}$ as $p \rightarrow \infty$.

and

$y^{\prime}(x) \geq k-\left[\int_{0}^{x} \frac{k_{1} s y_{l p}(s)+k\left(1-s^{2}\right) y^{\prime}(s)}{y_{l p}^{2}(s)} d s\right]$ on

$[0,1)$, where $y_{l p}(s)=k s^{4} \ln (2-s)+k s(1-s)^{2}$.

Now we show that $y \in C[0,1] \cap C^{2}[0,1)$.If we integrate $y_{p}^{\prime \prime}=\frac{-k_{1} x y_{p}-k\left(1-x^{2}\right) y_{p}^{\prime}}{y_{p}^{2}}$ from 0 to $x$, then we have

$y_{p}^{\prime}(x)-y_{p}^{\prime}(0)=-\int_{0}^{x} \frac{k_{1} \xi y_{p}(\xi)+k\left(1-\xi^{2}\right) y_{p}^{\prime}(\xi)}{y_{p}^{2}(\xi)} d \xi$

or,

$y_{p}^{\prime}(x)=k-\int_{0}^{x} \frac{k_{1} \xi y_{p}(\xi)+k\left(1-\xi^{2}\right) y_{p}^{\prime}(\xi)}{y_{p}^{2}(\xi)} d \xi .(2.2)$

If we integrate both sides of (2.2) from 0 to $x$, then we obtain

$y_{p}(x)-y_{p}(0)=k x-$

$\int_{0}^{x} \int_{0}^{s} \frac{k_{1} \xi y_{p}(\xi)+k\left(1-\xi^{2}\right) y_{p}^{\prime}(\xi)}{y_{p}^{2}(\xi)} d \xi d s$

Let

$$
u(s)=\int_{0}^{s} \frac{k_{1} \xi y_{p}(\xi)+k\left(1-\xi^{2}\right) y_{p}^{\prime}(\xi)}{y_{p}^{2}(\xi)} d \xi
$$

Then we have,

$y_{p}(x)-y_{p}(0)=k x-\int_{0}^{x} u(s) d s$ 


$$
\begin{aligned}
& =k x-x \int_{0}^{x} \frac{k_{1} \xi_{p}(\xi)+k\left(1-\xi^{2}\right) y_{p}^{\prime}(\xi)}{y_{p}^{2}(\xi)} d \xi+ \\
& \int_{0}^{x} s \frac{k_{1} s y_{p}(s)+k\left(1-s^{2}\right) y_{p}^{\prime}(s)}{y_{p}^{2}(s)} d s
\end{aligned}
$$

Changing $s$ to $\xi$ we have

$$
\begin{aligned}
& y_{p}(x)-y_{p}(0)= \\
& k x-x \int_{0}^{x} \frac{k_{1} \xi_{p}+k\left(1-\xi^{2}\right) y_{p}^{\prime}}{y_{p}^{2}(\xi)} d \xi+ \\
& \int_{0}^{x} \frac{k_{1} \xi^{2} y_{p}+k \xi\left(1-\xi^{2}\right) y_{p}^{\prime}}{y_{p}^{2}(\xi)} d \xi
\end{aligned}
$$

If we let $p \rightarrow \infty$ in both sides of (2.2) and (2.3), then by Lebesgue's Dominated Convergence Theorem, we obtain

$$
y^{\prime}(x)=k-\int_{0}^{x} \frac{k_{1} \xi y(\xi)+k\left(1-\xi^{2}\right) y^{\prime}(\xi)}{y^{2}(\xi)} d \xi .
$$

and

$$
\begin{aligned}
& y(x)-y(0)=k x-\int_{0}^{x} \frac{k_{1} \xi y+k\left(1-\xi^{2}\right) y^{\prime}}{y^{2}(\xi)} d \xi+ \\
& \int_{0}^{x} \frac{k_{1} \xi^{2} y+k \xi\left(1-\xi^{2}\right) y^{\prime}}{y^{2}(\xi)} d \xi,
\end{aligned}
$$

which implies that $y \in C^{2}[0,1)$. Since $y$ converges to 0 as $x$ approaches $1, y$ is continuous at $x=1$, which implies $y \in C[0,1] \cap C^{2}[0,1)$. Finally, we shall show that $y$ is a positive solution of (1.8). It is clear that $y^{\prime}(0)=k$ and $y(1)=0$.

If we take second derivatives on both sides of

$$
y^{\prime}(x)=k-\int_{0}^{x} \frac{k_{1} \xi y(\xi)+k\left(1-\xi^{2}\right) y^{\prime}(\xi)}{y^{2}(\xi)} d \xi
$$

we obtain

$$
y^{\prime \prime}(x)=-\frac{k_{1} x y(x)+k\left(1-x^{2}\right) y^{\prime}(x)}{y^{2}(x)},
$$

which implies that $y$ is a positive solution of (1.8).

Theorem 2.10: (Uniqueness): Assume that $y_{1}$ and $y_{2}$ are two positive solutions of (1.8). Then $y_{1}=y_{2}$.

Proof: The proof of this theorem is similar to that of Lemma 2.6.

\section{Conclusion}

Shin ${ }^{1}$ established the existence and uniqueness of a positive solution of (1.9) by using the method of upper and lower solutions. In this article we also established the existence and uniqueness of a positive solution of (1.8) by using the method of upper and lower solutions. Equation (1.8) and (1.9) are very different in form and physically because equation (1.8) represents decelerating flow and equation (1.9) represents Homann flow. The positive lower and upper solutions of (1.8) found in this article are achievement of us. The establishment of the existence and uniqueness of a positive solution of (1.8) using the constructive method such as the method of upper and lower solutions is also an achievement of us. We hope that the result obtained in this article will be useful to compare the numerical solution of (1.8).

1. Jun Yong Shin, 1997. A singular nonlinear differential equation arising in the Homann flow, J.Math. Anal. Appl. 212, 443-451.

2. Shanti Swarup, 2000, Fluid Dynamics, Krishna Prakashan Media (p) Ltd. Merut.

3. K. Schmidt, 1970. A nonlinear boundary value problem, J. Differential Equations 7, 527-537. 